\title{
Differentially Testing Soundness and Precision of Program Analyzers
}

\author{
Christian Klinger \\ Saarland University, Germany \\ io@klinch.de
}

\author{
Maria Christakis \\ MPI-SWS, Germany \\ maria@mpi-sws.org
}

\author{
Valentin Wüstholz \\ ConsenSys, Germany \\ valentin.wustholz@consensys.net
}

\begin{abstract}
In the last decades, numerous program analyzers have been developed both by academia and industry. Despite their abundance however, there is currently no systematic way of comparing the effectiveness of different analyzers on arbitrary code. In this paper, we present the first automated technique for differentially testing soundness and precision of program analyzers. We used our technique to compare six mature, state-ofthe art analyzers on tens of thousands of automatically generated benchmarks. Our technique detected soundness and precision issues in most analyzers, and we evaluated the implications of these issues to both designers and users of program analyzers.
\end{abstract}

\section{INTRODUCTION}

Program analyzers are very effective in detecting code issues and, especially in recent years, they are increasingly applied in industry to detect defects in real-world software. Notable examples of such analyzers are Facebook's Infer [1], [2], which is used to find resource leaks and null-pointer exceptions in Android and Java applications, Microsoft's SAGE [3], which is credited to have detected about one third of all security bugs that were discovered with fuzzers during the development of Windows 7 [4], and AbsInt's Astrée [5], [6], which can prove the absence of runtime errors and invalid concurrent behavior in safety-critical software, such as that of Airbus.

Despite the abundance of program analyzers that have been produced both by academia and industry, there is currently no systematic way of comparing their effectiveness on arbitrary code. To compare the soundness and precision of a set of analyzers, one could try them on a number of programs to get a feel for their false positive or false negative rates. However, just classifying the generated warnings as false or true positives would require considerable human effort, let alone determining whether any bugs are missed.

Alternatively, one could rely on the outcome of software verification competitions such as SV-COMP [7], which compares program analyzers based on an established collection of verification tasks. Although verification competitions are extremely valuable in increasing the visibility of new analyzers, providing a discussion forum for state-of-the-art techniques, and maintaining a set of programs with explicit properties to be checked, the verification tasks are rather stable. As a consequence, program analyzers can be designed to perform well in such competitions by specifically tailoring their techniques to the given benchmarks. In addition, the explicit checks might not be representative of properties in real-world software.
Our approach. To address these issues, we present the first automated technique for differentially testing program analyzers on arbitrary code. Given a set of seed programs, our approach automatically generates program-analysis benchmarks and compares the soundness and precision of the analyzers on these benchmarks. As a result, the effectiveness of the different program analyzers is evaluated in a systematic and automated way, the benchmarks are hardly predictable, and the explicit checks can be parameterized to express several types of properties, for instance, numerical, non-nullness, or points-to properties.

However, as for existing differential testing techniques, it is challenging to automatically derive the ground truth, for example, which warnings are indeed true positives or which errors are missed. We address this challenge by leveraging Engler et al.'s "bugs-as-deviant-behavior" strategy [8]. Specifically, when most program analyzers agree that a certain property does not hold, our approach detects a potential soundness issue in the deviant analyzers, which find that the property does hold. Conversely, we detect a potential precision issue when a few analyzers claim that a property does not hold, while the majority of analyzers verify the property.

The work most closely related to ours is by Kapus and Cadar, who use random program generation and differential testing to find bugs in symbolic execution engines [9]. In contrast to this work, our approach focuses on detecting soundness and precision issues in any program analyzer, potentially including a test generator based on symbolic execution. Moreover, our technique automatically generates programanalysis benchmarks from a given set of seed programs, possibly containing code that is difficult to handle by program analysis. In general, it is very challenging to randomly generate programs from scratch such that they reveal soundness and precision issues in mature analyzers, which is why our technique leverages the seed programs.

Overall, we expect our approach to guide users in making informed choices when selecting a program analyzer. However, this is not to say that the best analyzer is the most sound; users have varying needs depending on how critical the correctness of their code is and where in the software development cycle they are (e.g., before a code review or product release) [10]. We also expect our technique to assist designers of analyzers in detecting soundness and precision issues of their implementation, and to help enrich the collection of tasks 
used in verification competitions by automatically generating challenging, yet less predictable, benchmarks.

Contributions. We make the following contributions:

- We present the first automated technique for differentially testing soundness and precision of program analyzers on arbitrary code.

- We implement this technique in a tool architecture that compares analyzers on $\mathrm{C}$ programs and may be instantiated with any program analyzer for $\mathrm{C}$.

- We used our technique to compare six state-of-the-art program analyzers on about 26,000 programs. We found soundness and precision issues in four (out of six) analyzers, and we evaluated their significance to both program-analysis designers and users.

Terminology. Since terminology varies across different program-analysis techniques, we introduce the following terms that we use throughout the paper. Sound program analysis over-approximates the set of possible executions in a given program in order to prove the absence of errors in the program. Due to its over-approximation, sound analysis may generate false positives, that is, spurious warnings about executions that are not erroneous or even possible in the program. In contrast, a true positive is a warning about an actual error in the program. Unsound program analysis may over-approximate certain program executions and under-approximate others in order to find bugs, rather than prove their absence. In case an unsound analysis fails to detect an error in the program, we say that it generates a false negative.

An imprecise program analysis abstracts certain program executions such that it considers more executions than those feasible in the program. Although an imprecise analysis might generate false positives, it is not necessarily sound. On the other hand, a precise program analysis uses abstractions that closely describe executions in the program to generate as few false positives as possible.

Outline. The next section gives an overview of our approach through a running example. Sect. III explains the technical details of our approach, while Sect. IV describes our implementation. We present our experimental evaluation and threats to the validity of our results in Sect. V. We discuss related work in Sect. VI and conclude in Sect. VII.

\section{OVERVIEW}

In this section, we illustrate the workflow and tool architecture of our differential testing technique for program analyzers, shown in Fig. 1.

Workflow. Our technique, which is implemented in a tool called $\alpha$-Diff (pronounced "alpha-diff"), takes as input one or more correct seed programs that do not contain any (explicit or implicit) assertions. Because these programs are both correct and assertion-free, program analyzers (even if sound and imprecise) do not generate any warnings for them.

Next, $\alpha$-Diff parses a seed program, and based on one of its search strategies (Sect. III-A), a program location is selected. At this location, $\alpha$-Diff synthesizes and introduces a check, in the form of an assertion, expressing a property of interest

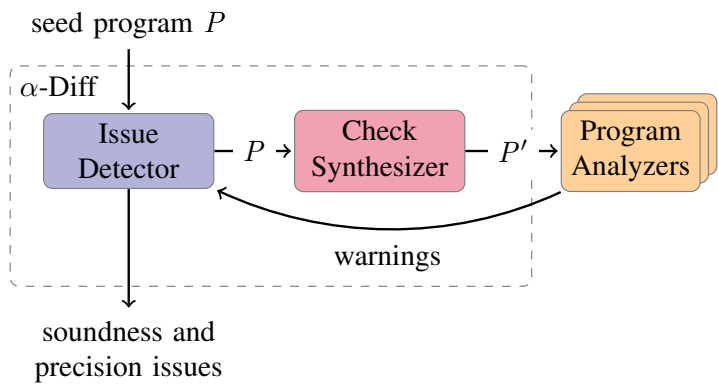

Figure 1: Overview of the workflow and tool architecture.

(e.g., a numerical property) and involving variables that are in scope at the program location (Sect. III-A). We call the resulting program a variant of the seed program.

On this program variant, which now contains a single assertion, $\alpha$-Diff runs a set of program analyzers. The results of the analyzers, that is, the presence or absence of any generated warnings for the assertion, are recorded.

Subsequently, $\alpha$-Diff selects a new program location in the same seed program and repeats the process until a given budget (i.e., number of synthesized checks for a particular seed program) is depleted. The tool then continues to parse another seed program, if any.

When all seed programs have been instrumented and analyzed until the budget, $\alpha$-Diff compares the recorded results and reports any detected soundness and precision issues in the analyzers (Sect. III-B).

Example. Fig. 2 shows a simplified version of an SV-COMP benchmark that we used in our experimental evaluation as a seed program to test six program analyzers. (Lines 4 and 7 should be ignored for now.) Note that this program is correct and does not contain any assertions. Therefore, when running all analyzers on this code, no warnings are generated.

When passing this seed program to $\alpha$-Diff, the 'Check Synthesizer' (from Fig. 1), which is implemented to synthesize checks for numerical properties, introduces the assertion on line 4. Our tool then runs all analyzers on the resulting program variant, whose assertion can obviously fail. All analyzers detect the assertion violation except for CBMC [11], a bounded model checker for $\mathrm{C}$ and $\mathrm{C}++$ programs, and SMACK [12], a software verifier that translates the LLVM intermediate representation into the Boogie intermediate verification language [13]. CBMC typically unrolls loops as many times as necessary such that all bugs are found, but we imposed a time limit on all analyzers, which proved to be insufficient for CBMC to unroll the loop enough times such

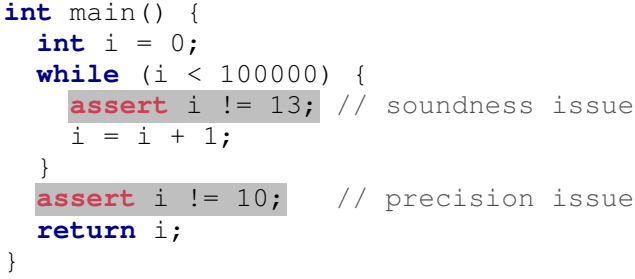

Figure 2: Soundness and precision issues in SMACK. 
that the assertion violation is detected. (Note, however, that CBMC does find the bug with a higher time limit.) Still, CBMC soundly returns 'unknown', that is, no bugs were found but, due to reaching the time limit, the program has not been verified. On the other hand, SMACK claims to have verified the assertion on line 4, which indicates a soundness issue.

We reported this issue ${ }^{1}$ to the designers of SMACK, who told us that the assertion violation is missed due to a sizereduction heuristic, which searches for large constants in SVCOMP benchmarks, such as 100000 in Fig. 2, and replaces them with smaller numbers, in our case with 10 , to reduce the benchmark size. This heuristic is unsound and specifically tailored to the competition benchmarks.

In addition to being a source of unsoundness, this heuristic can also be the cause of imprecision. For example, consider the program variant of Fig. 2 with the assertion on line 7 (and without the assertion on line 4). This assertion is introduced by $\alpha$-Diff within the budget that is assigned to the seed program from SV-COMP, and it can never fail. All analyzers but SMACK are able to verify this program. However, because of the size-reduction heuristic, SMACK knows that variable $i$ is equal to 10 right after the loop, and therefore, the verifier reports an assertion violation, indicating a precision issue.

\section{Differential Testing of Program Analyzers}

In this section, we describe the main components of our workflow in more detail and explain precisely how we detect soundness and precision issues in program analyzers.

\section{A. Check Synthesis}

The check-synthesis component consists of two aspects: (1) the instrumentation, which creates a check and introduces it at a certain location in the seed program to generate a variant, and (2) the search strategies, which explore the space of possible seed-program variants that may be generated. We discuss these aspects next.

Instrumentation. Alg. 1 describes how $\alpha$-Diff generates a variant $P^{\prime}$ from a seed program $P$. First, the algorithm selects a candidate expression $e$ at program location $l$ in $P$. In our context, a candidate expression is a pure expression of integral type that reads from at least one variable, e.g., $c+3$ or $a[i]$, where $c$ is a variable of type char and $a$ is an array of integers. (Note that the choice of expression $e$ is made according to a search strategy.) Next, the algorithm generates a constant $k$, which is used, together with expression $e$, to create an assertion of the form assert $e !=k$. To generate variant $P^{\prime}$, this assertion is inserted at location $l$ in $P$. As an example, consider the assertion on line 4 of Fig. 2, which is introduced at the program location where expression $i$ (righthand side of the assignment on line 5) is found in seed program $P$. Similarly, the assertion on line 7 is inserted at the location of the return statement in $P$.

The assertions introduced by $\alpha$-Diff in a seed program check numerical properties. In particular, they check whether

\footnotetext{
$1_{\text {https://github.com/smackers/smack/issues/324 }}$
}

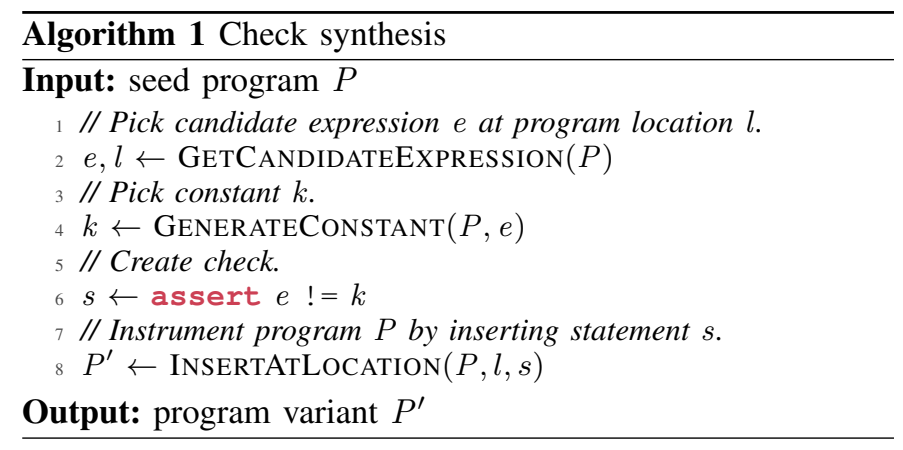

candidate expression $e$ can ever have value $k$ at program location $l$. If so, then the assertion can fail, and the tested analyzers should detect this violation in order to be sound. If the expression can never have this value at that location, then the assertion cannot fail, and the analyzers should not detect any violation in order to be precise.

Note that, although $\alpha$-Diff generates program variants that check numerical properties, the 'Check Synthesizer' of Fig. 1 is configurable and may be extended to also synthesize other types of properties. Still, as we discuss in Sect. V-C, numerical properties were sufficient in detecting soundness and precision issues in most of the analyzers we tested.

Batch checks. To reduce the number of times the program analyzers are invoked, $\alpha$-Diff can also synthesize assertions with multiple conjuncts, which we call 'batch checks'. For example, a program variant of Fig. 2 could check whether $i$ is ever equal to 10 or 11 on line 7 with the assertion assert $i \quad !=10 \& \& i \quad !=11$. Recall that, at this program location, SMACK knows that $i$ has value 10 and would, therefore, detect a violation only due to the first conjunct of the above assertion. For such cases, $\alpha$-Diff uses divideand-conquer to eliminate conjuncts that do not cause any disagreement between the program analyzers. We evaluate the effectiveness of our technique when synthesizing assertions with batch checks in Sect. V-C.

Search strategies. In addition to generating a value $k$, Alg. 1 also explores the search space of possible candidate expressions $e$. Our technique navigates this space using a number of static and dynamic search strategies, which we describe below and evaluate in Sect. V-C. Note that the search strategies are applied by the GETCANDIDATEEXPRESSION function in Alg. 1, which is configurable in our implementation.

Static strategies. Static strategies traverse the abstract syntax tree (AST) of the seed program to collect all the candidate expressions. These strategies then compute a weight $w_{e}$ for each candidate expression $e$ (according to a weight function), sum all weights to compute the total $w_{t}$, and assign to each expression $e$ the probability $w_{e} / w_{t}$ of being selected by function GetCAndidATEEXPRESSION. Overall, $\alpha$-Diff supports three static strategies that differ in their weight functions:

- The Uniform-Random strategy selects candidate expressions uniformly. In other words, all possible locations have a weight of 1 . 
- The Breadth-Biased strategy assigns to each candidate expression at location $l$ a weight of $1 / \operatorname{depth}(l)$, where depth $(l)$ is the depth of location $l$ in the AST. This means that this strategy assigns larger weights to locations higher in the AST.

- The Depth-Biased strategy assigns to each candidate expression at location $l$ a weight of $\operatorname{depth}(l)$, that is, larger weights are assigned to locations lower in the AST.

Dynamic strategies. Dynamic strategies do not assign fixed weights to the candidate expressions. Instead, these strategies select an initial expression and then traverse the AST in different directions to select another. Our tool supports the following two dynamic strategies that differ in how they traverse the AST:

- The Random-Walk strategy selects an initial candidate expression at the first possible location in the main function of the seed program. To select another expression, this strategy moves in a random direction in the AST, for instance, to the subsequent statement, the previous compound statement, or into a function call.

- The Guided-Walk strategy is a variation of RandomWalk. In comparison, this strategy favors moves to locations in the AST that are likely to increase differences in the running times of the program analyzers, for example, by moving deeper in a compound statement.

\section{B. Detection of Soundness and Precision Issues}

A common challenge for differential testing techniques is detecting issues with a low false-positive rate, instead of reporting all found differences. In our context, this requires determining whether the analysis results are indeed sound or precise and, in particular, whether any generated warnings are spurious or miss errors in the program. To address this challenge, $\alpha$-Diff uses two mechanisms for detecting soundness and precision issues in the tested analyzers, namely, the deviance and unsoundness detection mechanisms.

Deviance detection. Given a program variant (with a single assertion), analyzers return one of the following verdicts: safe (i.e., the assertion cannot fail), unsafe (i.e., the assertion can fail), or unknown (i.e., it is unknown whether the assertion can fail, likely because the analysis times out).

The deviance detection mechanism is inspired by Engler et al.'s "bugs as deviant behavior" [8]. Specifically, given a program variant, when the majority of program analyzers return unsafe, $\alpha$-Diff detects a potential soundness issue in the deviant analyzers that return safe. Conversely, $\alpha$-Diff detects a potential precision issue when a few analyzers return unsafe, while the majority of analyzers return safe.

We call an analyzer $\delta$-unsound with respect to a program variant when it returns safe and $\delta$ other analyzers return unsafe. Analogously, an analyzer is $\delta$-imprecise with respect to a variant when it returns unsafe and $\delta$ other analyzers return safe. For each tested analyzer, $\alpha$-Diff ranks all detected soundness (resp. precision) issues in order of decreasing severity, where severity is proportional to $\delta$, that is, to the number of disagreeing analyzers. For instance, for the program variant of
Fig. 2 containing the assertion on line 7, we say that SMACK is 5-imprecise since all other analyzers disagree.

Unsoundness detection. Certain analyzers underapproximate the set of possible executions in a given program. Consequently, when such analyzers detect an error in the program, this is inevitably a true positive (modulo bugs in the analysis itself). We consider CBMC to be an under-approximating program analyzer, for instance, because it typically analyzes the program until a bound is reached and uses bit-precise reasoning (i.e., no over-approximation).

When such analyzers (like CBMC) find that a program variant is unsafe, then the assertion in the variant can definitely fail. Therefore, any other analyzer that returns safe for the same variant is unsound, and we call it must-unsound. On the other hand, when CBMC returns safe, it is possible that it has missed an assertion violation in the program variant due to its under-approximation. For this reason, we do not use the results of under-approximating analyzers to draw any definite conclusions about imprecision in other analyzers.

\section{IMPLEMENTATION}

In this section, we present the details of our implementation, which is open source ${ }^{2}$.

Check synthesis. Recall from Alg. 1 that the checksynthesis component of $\alpha$-Diff generates a constant $k$, which is used, together with a candidate expression, to form a check for a numerical property. In general, the constants $k$ are sampled randomly but with a few tweaks.

To guide the sampling, our implementation uses the following optimizations. First, while parsing a seed program, $\alpha$-Diff populates a pool of constants with any constant $c$ encountered in the program, $c+1, c-1$, and boundary values (such as $M I N_{-} I N T, 0$, and $\left.M A X_{-} I N T\right)$. The constants in the pool are then used to complement the randomly sampled constants. Second, our implementation does not sample constants uniformly to avoid frequently generating very large values. Instead, $\alpha$-Diff uniformly selects a bit-width for a constant and then randomly generates a sequence of bits with this width. Third, for each check to be synthesized, we use a type checker to determine the type of the candidate expression and, thus, the bit-width of the corresponding constant that will be generated (e.g., 1 bit for expressions of type bool and 8 bits for char).

All these optimizations aim to bias or restrict the search space of possible constants and, therefore, improve the efficiency of our tool.

Program analysis runs. After invoking the program analyzers, our implementation persists the results of each analysis run in a database. The database is extended with caching capabilities allowing $\alpha$-Diff to avoid re-running the analyzers on program variants that have been previously generated.

Moreover, incorporating new program analyzers in $\alpha$-Diff is very easy since our implementation uses Docker containers to install, set up, and run the analyzers. This also greatly simplifies resource management and monitoring (e.g., memory

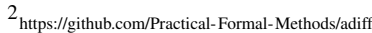


and CPU usage, or running time) as well as the parallelization of analysis jobs.

Issue detection. To detect soundness or precision issues, users can query the database of analysis results, either by writing their own queries or by using the default ones (described in Sect. III-B). A query may be submitted either through the command line or via a web-based user interface.

\section{EXPERIMENTAL EVALUATION}

To evaluate the effectiveness of our approach, we apply $\alpha$-Diff to six state-of-the-art program analyzers. In this section, we present our experimental setup (Sect. V-A), give an overview of the tested analyzers (Sect. V-B), and investigate several research questions (Sect. V-C).

\section{A. Setup}

We selected 1,393 seed programs (written in C) from the SV-COMP repository of verification benchmarks [7]. We excluded all programs that contain "float", "driver", or "ecarers2012" in their path. The first category of excluded programs cannot be handled by all program analyzers we tested, while the other two categories mainly contain very large benchmarks that caused most analyzers to reach the time limit we set for our experiments. We also excluded any other benchmark that crashed our type checker, for instance, some of the programs that are automatically generated by PSYCO [14].

We chose these benchmarks as seed programs because five out of the six program analyzers we tested have participated in at least one SV-COMP over the years. We were, therefore, confident that the analyzers would be able to handle most of the selected programs. Moreover, SV-COMP benchmarks typically do not exhibit arithmetic overflows to avoid penalizing analyzers that are intentionally unsound with respect to overflow [15], [16].

In general, all SV-COMP benchmarks are annotated with assertions (no other crashes should be possible), and user inputs are made explicit. To use these benchmarks as seed programs, $\alpha$-Diff had to preprocess them. First, we removed all existing assertions such that the seed programs are correct and the program analyzers do not generate any warnings for them. Second, we ran the GCC preprocessor to eliminate any macro usages and, thus, avoid any parsing issues in the analyzers.

Unless stated explicitly, we used the following default configuration of $\alpha$-Diff: a time limit of 30s, 2 CPU cores, and up to 8GB of memory per analysis run, a budget of either 100 or $20 \%$ of the number of candidate expressions (whichever is smaller) per seed program, the Uniform-Random search strategy, and a batch-check size of 4. Recall from Sect. III-A that $\alpha$-Diff can synthesize assertions with multiple conjuncts. These are called batch checks, and we refer to the number of conjuncts as the 'batch-check size'.

We ran our experiments on a dual hexacore

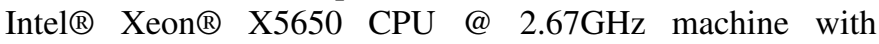
$48 \mathrm{GiB}$ of memory, running Debian Stretch.

\section{B. Program Analyzers}

We selected the analyzers under test such that they cover a wide range of different analysis techniques. In addition, we only chose mature tools that are under active development. We give a short description of each analyzer below. Note that, unless otherwise stated, we used their default configuration.

CBMC. CBMC [11] is a bit-precise bounded model checker that unrolls loops and expresses verification conditions as SMT queries over bit-vectors. We used version 5.3 of the tool.

CPAchecker. CPAchecker [17] is a software model checker that incorporates different program-analysis techniques, such as predicate abstraction [18], [19], lazy abstraction [20], and k-induction [21]. We used the development version $51.7-\operatorname{svn} 28636$ of the tool, which incorporates fixes for two soundness issues that $\alpha$-Diff detected. Note that CPAchecker won the first place in SV-COMP' 18.

Crab. Crab [22], [23] is an abstract interpreter that supports several abstract domains [24], [25]. Its default configuration uses the Zones domain [26], and we enabled inter-procedural analysis. The tool was built from commit 5dd7a00b5b.

SeaHorn. SeaHorn [27] is a software model checker that expresses verification conditions as Horn-clauses and uses existing solvers to discharge them. Its default configuration uses Spacer [28], [29], which is a fork of Z3 [30] with a variant of the IC3/PDR [31] model-checking algorithm for solving verification conditions. The tool was built from commit 59c4a917a595.

SMACK. SMACK [12] is software model checker that translates C programs to Boogie [13], which can be checked by a number of different verification back-ends. We used version 1.9 with the default configuration, which runs the Corral verifier [32]. We also enabled the svcomp extension and set the loop-unrolling bound to 1,000. Note that SMACK won the second place in SV-COMP' 17.

Ultimate Automizer. Ultimate Automizer (or UAutomizer) is a software model checker that uses an automata-based verification approach [33], [34], [35]. We used version 0.1.23. Note that UAutomizer won the second place in SV-COMP'18.

For all program analyzers that support this, we set the machine architecture to 32-bit. We also provided a default LTL-specification file to any analyzer that requires an explicit reachability property for checking assertions.

\section{Results}

We break our experimental results down into five categories, each investigating a different research question.

RQ1: Does $\alpha$-Diff find soundness and precision issues? Given as input the 1,393 seed programs, $\alpha$-Diff generated 25,960 program variants. Fig. 3 shows the number of potential soundness issues that $\alpha$-Diff detected in the tested program analyzers when running them on the generated variants. Each soundness issue corresponds to a program variant that revealed an analyzer to be must- or $\delta$-unsound. As shown in the figure, our technique detected a significant number of potential issues in four program analyzers. Note that some of these issues 


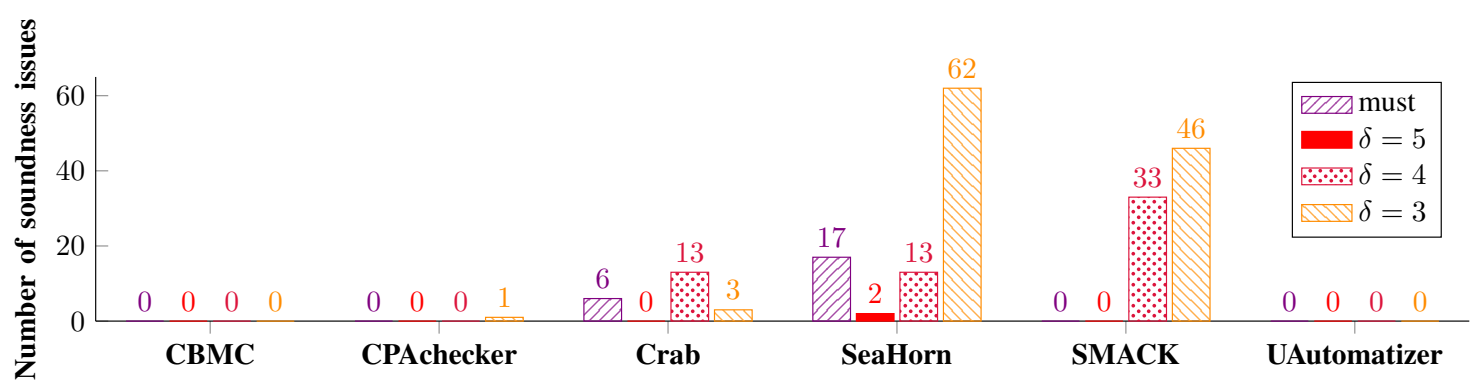

Figure 3: Soundness issues detected for each program analyzer.

might expose the same source of unsoundness in an analyzer. For example, assume that $\alpha$-Diff generates several variants that use bitwise arithmetic in their assertions. For each of these variants, our tool could potentially report a soundness issue in any analyzer that does not support bit-precise reasoning.

We manually inspected all detected issues from Fig. 3 and reported unique sources of unsoundness to the designers of Crab, SeaHorn, and SMACK. Note, however, that $\alpha$-Diff had previously found two soundness issues in CPAchecker, which were reported to the tool designers early on and were fixed $^{34}$. We used the patched version of CPAchecker for our experiments. We discuss the reaction of all designers in RQ2.

As shown in the figure, CPAchecker is found to be 3unsound for one program variant. This issue is actually a source of imprecision in three other analyzers, namely, in Crab, SeaHorn, and SMACK, and is related to bit-precise reasoning. In general, we observed that the false-positive rate of our technique depends on two main factors. First, if some of the tested program analyzers are imprecise for a given variant, $\alpha$-Diff detects soundness issues in the remaining analyzers, which are, however, sound. Second, any issues that are reported for $\delta$-unsound analyzers, where $\delta$ is small, are likely false positives. For example, when a program analyzer is 1-unsound, there exists only one disagreeing analyzer. In fact, inspecting issues found for $\delta$-unsound analyzers, where $\delta$ is large, significantly reduces the number of false positives caused by imprecision in some of the other analyzers. In the results of Fig. 3, we did not find any false positives when $\delta \geq 4$ or when inspecting the issues that were detected for must-unsound analyzers.

Fig. 4 shows the number of precision issues that $\alpha$-Diff found for the same program variants. Although the number of issues is significant, the majority of these do not correspond to bugs in the analyzers. Instead, most of the precision issues are either intended by the analysis designers (for instance, imprecise reasoning about numeric types) or inherent to the analysis (for example, imprecision in non-relational abstract domains, such as Intervals).

Overall, $\alpha$-Diff found many more precision issues in Crab in comparison to the other analyzers. These issues, however, are intended since Crab favors performance over precision, similarly to several other abstract interpreters. Manual inspec-

\footnotetext{
3 https://groups.google.com/d/msg/cpachecker-users/3JCOeNuoleA/fpr8ElaaBgAJ

4 https://groups.google.com/d/msg/cpachecker-users/3JCOeNuoleA/YDT7LokZBwAJ
}

tion of a selection of these issues showed that the program variants for which Crab is imprecise exhibited at least one of the following features: (1) usage of pointers, (2) bitwise operations, (3) invariants expressing parity of variables.

In addition to detecting issues in program analyzers, $\alpha$ Diff can also compare their relative soundness and precision. To determine the relative precision (resp. soundness) of an analyzer $A_{i}$ with respect to another analyzer $A_{j}$, our technique computes the probability that $A_{i}$ returns safe (resp. unsafe) given that $A_{j}$ returns safe (resp. unsafe). Tab. 1 shows these probabilities for all analyzers under test. Note that $<1.00$ stands for a probability between 0.99 and 1.00 .

From the first row of the table, we observe that CBMC verifies only $1 \%$ of the variants that all other analyzers prove safe. This is due to the fact that CBMC uses bounded model checking, which might fail to explore all program paths within a certain time limit. In contrast, CPAchecker verifies $71 \%$ of the variants that CBMC proves safe. As another example, SeaHorn verifies almost all variants that UAutomizer proves safe, while UAutomizer verifies only $81 \%$ of the variants that SeaHorn proves safe. This clearly indicates that SeaHorn is more precise on the generated program variants. On the other hand, $\alpha$-Diff did not detect any soundness issues for UAutomatizer (see Fig. 3), which could potentially explain the higher precision of SeaHorn on some variants. In general, analyzers might gain in precision when sacrificing soundness since they consider fewer program executions.

Tab. 2 shows a direct comparison between SMACK and Crab, which implement very different analysis techniques. Note that $>0 \%$ stands for a percentage between 0 and 1 .

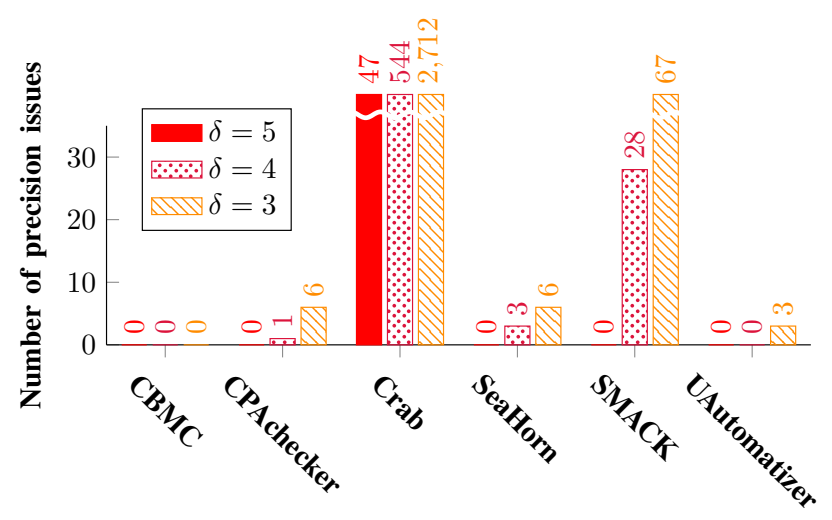

Figure 4: Precision issues detected for each analyzer. 


\begin{tabular}{|l|c|c|r|c|c|c|}
\hline $\boldsymbol{A}_{\boldsymbol{i}}$ & CBMC & CPAchecker & Crab & SeaHorn & SMACK & UAutomatizer \\
\hline CBMC & 1.00 & 0.01 & 0.01 & 0.01 & 0.01 & 0.01 \\
CPAchecker & 0.71 & 1.00 & 0.99 & 0.95 & 0.97 & 0.99 \\
Crab & 0.37 & 0.63 & 1.00 & 0.62 & 0.88 & 0.68 \\
SeaHorn & 0.77 & 0.98 & $<1.00$ & 1.00 & 0.99 & $<1.00$ \\
SMACK & 0.72 & 0.53 & 0.76 & 0.53 & 1.00 & 0.61 \\
UAutomatizer & 0.57 & 0.82 & 0.89 & 0.81 & 0.93 & 1.00 \\
\hline
\end{tabular}

Table 1: Relative precision for the tested program analyzers.

\begin{tabular}{|l|c|c|c|}
\hline SMACK Crab & unsafe & unknown & safe \\
\hline unsafe & $14 \%$ & $22 \%$ & $>0 \%$ \\
unknown & $30 \%$ & $0 \%$ & $7 \%$ \\
safe & $3 \%$ & $0 \%$ & $23 \%$ \\
\hline
\end{tabular}

Table 2: Comparison of results for SMACK and Crab.

As shown in the table, for 3\% of the program variants, Crab reports unsafe whereas SMACK returns safe. Both tools prove $23 \%$ of the variants safe and never return unknown for the same program variant. This suggests that these analysis techniques have complementary strengths and weaknesses.

In addition to comparing the results of different analyzers, $\alpha$-Diff can also be used to compare different configurations of the same analyzer. Tab. 3 shows a direct comparison of two Crab configurations, each using a different abstract domain, namely, Octagons [36] and Polyhedra [37]. As shown in the table, there is a small number of program variants that are verified with the Octagons domain but not with Polyhedra, although in theory Polyhedra is strictly more precise than Octagons. As pointed out by the designer of $\mathrm{Crab}^{5}$, this mismatch is due to the fact that the domains use different widening operations [38], [39] to speed up convergence of the fixed-point computation. This is a known caveat [40] and was independently evaluated in a recent paper comparing different abstract domains [41].

In Tab. 4, we use $\alpha$-Diff to compare the relative precision of several abstract domains of Crab, namely, Intervals, Octagons, Polyhedra, RTZ (i.e., the reduced product of disjunctive Intervals and Zones), and Zones. Across the domains, the differences in precision are small for the generated variants. However, not surprisingly, the Intervals domain is typically less precise than the others. For instance, Intervals can only verify $89 \%$ of the variants that are proved safe with Zones. On the other hand, the very precise Polyhedra domain can only verify $99 \%$ of the variants that are proved safe with Intervals. As previously explained, this is due to the widening operation.

RQ2: Are the issues relevant for designers of analyzers? To determine whether the issues that $\alpha$-Diff reports are relevant to analysis designers, we inspected all detected soundness issues as well as a selection of precision issues. Overall, we found ten unique soundness and precision issues in four (out of six) program analyzers (excluding CBMC and UAutomizer). We reported nine of these issues to the designers of the four analyzers. All reported issues were confirmed and three (in CPAchecker and Crab) were fixed in only a few hours each. We now discuss the detected issues in detail.

CPAchecker. Although for the experiments in this paper we used the patched version of CPAchecker, $\alpha$-Diff detected two

\footnotetext{
$5_{\text {https://github.com/seahorn/crab-llvm/issues/18 }}$
}

\begin{tabular}{|l|r|r|c|}
\hline oct pk & unsafe & unknown & safe \\
\hline unsafe & $43 \%$ & $1 \%$ & $>0 \%$ \\
unknown & $>0 \%$ & $10 \%$ & $>0 \%$ \\
safe & $>0 \%$ & $>0 \%$ & $45 \%$ \\
\hline
\end{tabular}

Table 3: Comparison of Crab Octagons and Polyhedra.

sources of unsoundness in this analyzer, which were immediately fixed. The first soundness issue ${ }^{6}$ was revealed in the simplified version of a program variant shown in Fig. 5 (line 4 should be ignored). In this program, $\mathrm{x}$ is assigned a nondeterministic integer and, therefore, the assertion on line 3 can fail. A previous version of CPAchecker missed this assertion violation due to a bug in its invariant-generation component, which was unsound when trying to obtain information about the factors of a multiplication whose product was zero.

The second issue ${ }^{7}$ was revealed in the simplified variant of Fig. 5 when considering line 4 (and ignoring line 3). According to the $\mathrm{C}$ standard, the expression $\mathrm{x}==1$ evaluates to an integer of value 0 or 1 , which is never equal to 99 . Consequently, the assertion on line 4 can fail since $\mathrm{x}$ is assigned a non-deterministic integer. CPAchecker missed this assertion violation due to a bug in its value analysis, which was unsound when analyzing nested binary expressions such as the property asserted above.

Crab. Our technique detected two soundness issues in Crab. The first issue ${ }^{8}$ made the inter-procedural analysis of Crab unsound in the presence of recursion, and the bug was immediately fixed.

The second issue (which was reported together with the first) is caused by Crab's LLVM-based [42] front-end, which may optimize the program by exploiting undefined behavior. For example, several seed programs contain uninitialized variables. According to the $\mathrm{C}$ standard, the behavior of a program that reads from such variables is undefined, that is, any behavior is correct. In these cases, a compiler pass may under-approximate the behavior of the program, for instance, by assuming that any read from an uninitialized variable returns 0 , to optimize the executable code. However, this under-approximation potentially leads to unsoundness in program analyzers that analyze the optimized code, given that the original program may fail when compiled without this optimization or with a different compiler.

We also reported two imprecision issues ${ }^{9}$ in the Polyhedra and Octagons domains of Crab. In particular, the less precise Intervals domain was able to verify the assertion in a program

\footnotetext{
$6_{\text {https://groups.google.com/d/msg/cpachecker-users/3JCOeNuoleA/fpr8ElaaBgAJ }}$

7 https://groups.google.com/d/msg/cpachecker-users/3JCOeNuoleA/YDT7LokZBwAJ

8 https://github.com/seahorn/crab-llvm/issues/20

9 https://github.com/seahorn/crab-llvm/issues/18
} 


\begin{tabular}{|c|c|c|c|c|c|}
\hline$\widehat{D_{i}} D_{j}$ & int & oct & pk & rtz & zones \\
\hline int & 1.00 & 0.97 & 0.97 & 0.97 & 0.89 \\
\hline oct & 0.99 & 1.00 & 0.99 & 0.99 & 0.99 \\
\hline pk & 0.99 & 0.99 & 1.00 & 0.98 & 0.99 \\
\hline rtz & $<1.00$ & $<1.00$ & 0.99 & 1.00 & $<1.00$ \\
\hline zones & 1.00 & $<1.00$ & 0.99 & 0.99 & 1.00 \\
\hline
\end{tabular}

Table 4: Relative precision for abstract domains of Crab.

variant, whereas the more precise Polyhedra domain found the variant unsafe. As discussed in RQ1, such precision issues are possible for abstract domains with a widening operation. The issue was similar for the Octagons domain, which typically ignores dis-equalities. In contrast, given the interval $x=[0,10]$ and the constraint $x \neq 10$, the Intervals domain does compute the more precise interval $x=[0,9]$.

SeaHorn. We reported a soundness ${ }^{10}$ and a precision issue ${ }^{11}$ to the designers of SeaHorn, who confirmed both issues. The soundness issue was caused by SeaHorn's LLVM-based frontend, which is slightly different than Crab's and, thus, results in different unsound results.

Regarding the precision issue, the designers of SeaHorn explained that it is due to the conservative handling of bitwise operations and numeric types. In particular, all numeric types are abstracted into arbitrary-precision signed integers.

$S M A C K$. We reported the soundness and precision issues ${ }^{12}$ that are caused by the size-reduction heuristic in SMACK (see Sect. II for details). Although confirmed, these issues were not fixed since this behavior was intended by the designers.

We also found several other soundness issues, which were due to optimizations by SMACK's LLVM-based front-end, just like in Crab and SeaHorn. We did not report these issues to the designers since their cause is clear.

In general, the reaction of the analysis designers to all reported issues shows that $\alpha$-Diff can detect important sources of unsoundness and imprecision. This is especially the case since the tested analyzers are mature tools that are under active development. Moreover, five of these analyzers (excluding Crab) have participated in SV-COMP, which did not reveal any of the above bugs.

RQ3: Are the controversial issues relevant for users? Half of the tested program analyzers, namely Crab, SeaHorn, and SMACK, might be unsound in the presence of undefined behavior. As discussed earlier, this unsoundness is caused by compiler optimizations that under-approximate the behavior of the program. We call such soundness issues controversial because different compilers are inconsistent in reasoning about undefined behavior and, consequently, the results of analyzers that analyze executable code can be contradictory.

To shed more light on what users expect from program analyzers in the presence of undefined behavior, we performed a survey of 16 professional developers, who we hired on Upwork $^{13}$. To screen the candidates, we used two short interview questions (about type-conversion rules and pointer usage in

\footnotetext{
$10_{\text {https://github.com/seahorn/seahorn/issues/152 }}$

$11_{\text {https://github.com/seahorn/seahorn/issues/157 }}$

12 https://github.com/smackers/smack/issues/324

$13 \mathrm{https://www.upwork.com/}$
}

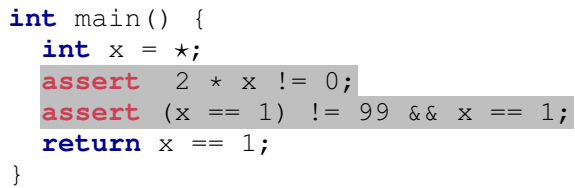

Figure 5: Soundness issues in CPAchecker.

C). Out of the candidates that replied correctly, we selected those that had experience with $\mathrm{C}$.

The survey contained 9 short tasks. Each task included a short $\mathrm{C}$ program, which was a simplified version of a program variant generated by $\alpha$-Diff. For every task, we asked whether the assertion in the given program can fail, and just like a program analyzer, a survey participant could respond with yes (i.e., unsafe), no (i.e., safe), and I don't know (i.e., unknown).

To pilot the survey tasks, we sent the survey to 4 students and interns who study Computer Science and already have a Bachelor's degree. We asked these participants if they found any portion of the survey difficult to understand and requested their feedback. Their responses were solely used to improve the survey.

After finalizing the tasks, we sent the survey to the professional developers. The tasks were presented to the developers in a randomized order, but in total, the survey included 6 non-controversial and 3 controversial questions. The noncontroversial questions asked about programs for which most analyzers were in agreement regarding their safety, whereas the controversial questions asked about unsafe programs that contained undefined behavior. We used the non-controversial tasks to exclude participants who gave the wrong answer to at least 4 (out of 6) of these questions. Based on this threshold, we excluded 4 (out of 16) survey participants.

Tab. 5 shows the survey responses from the 12 developers that we did not exclude. The first column shows the task identifier: tasks 1-6 are non-controversial and tasks 7-9 are controversial. Next to each task identifier, we indicate whether there exists an execution of the corresponding program that fails. For example, when the executable code of the programs in tasks 7-9 is not optimized, the assertions can be violated. The remaining columns of the table show the survey responses categorized as unsafe, unknown, and safe.

As shown in the table, the majority of the participants (10 out of 12 professional developers) considered the controversial programs to be unsafe. This clearly suggests that program analyzers should treat undefined behavior as non-determinism, instead of optimizing it away. On the other hand, the 4

\begin{tabular}{|c|c|c|c|}
\hline \multirow{2}{*}{$\begin{array}{c}\text { Task } \\
\text { Identifier }\end{array}$} & \multicolumn{3}{|c|}{ Survey Response } \\
\hline & unsafe & unknown & safe \\
\hline (unsafe) & 11 & 0 & 1 \\
\hline (unsafe) & 12 & 0 & 0 \\
\hline (unsafe) & 11 & 0 & 1 \\
\hline (safe) & 2 & 0 & 10 \\
\hline (safe) & 0 & 0 & 12 \\
\hline (safe) & 0 & 0 & 12 \\
\hline (unsafe) & 10 & 1 & 1 \\
\hline (unsafe) & 10 & 1 & 1 \\
\hline (unsafe) & 10 & 1 & 1 \\
\hline
\end{tabular}

Table 5: Survey responses from professional $\mathrm{C}$ developers. 


\begin{tabular}{|l|c|c|c|}
\hline Search Strategy & \multicolumn{3}{|c|}{ Number of Detected Issues } \\
& must-unsound & $\geq$ 3-unsound & $\geq$ 3-imprecise \\
\hline Uniform-Random & 2 & 2 & 71 \\
Breadth-Biased & 1 & 2 & 70 \\
Depth-Biased & 1 & 1 & 77 \\
Random-Walk & 0 & 0 & 59 \\
Guided-Walk & 1 & 2 & 39 \\
\hline
\end{tabular}

Table 6: Effect of search strategies on the number of detected issues.

excluded developers were not able to give correct answers to at least 4 questions from tasks $1-6$, and the remaining 12 developers gave 4 wrong answers to these tasks (see Tab. 5). This is a strong indication that even professional $\mathrm{C}$ developers benefit from program analysis.

RQ4: What is the effect of the search strategy? To generate a seed-program variant, our technique explores the search space of all possible candidate expressions using five different search strategies (see Sect. III-A). To evaluate how each search strategy affects the number of detected issues, we ran $\alpha$-Diff on 20 seed programs, which were randomly selected from the seed programs used in the evaluation of RQ1. For each run of $\alpha$-Diff on the seed programs, we enabled a different search strategy, and we measured the number of soundness and precision issues that were found. We used the default configuration of our tool, but with a batch-check size of 1 , to prevent batch checks from influencing the results.

Tab. 6 shows the effect of the five search strategies on the number of detected issues. The first column of the table shows the search strategy, the second the cumulative number of soundness issues detected in all must-unsound analyzers, the third the soundness issues detected in $\geq 3$-unsound analyzers (that is, in 3-, 4-, and 5-unsound analyzers), and the fourth the precision issues detected in $\geq 3$-imprecise analyzers. In general, the results suggest that the static search strategies are more effective in detecting soundness and precision issues than the dynamic strategies. Among the static strategies, the Uniform-Random strategy helps find the most soundness issues, although the differences are small. Among the dynamic strategies, the Random-Walk strategy performs the worst. We also observed that Breadth-Biased and Guided-Walk each detect a soundness issue that is not found by any other strategy.

RQ5: What is the effect of batch checks? To evaluate the influence of the batch-check size on the effectiveness of our approach, we ran $\alpha$-Diff on the same seed programs that were selected for the evaluation of RQ4. We used the UniformRandom search strategy, and otherwise, the same configuration of our tool as in the experiment of RQ4.

Tab. 7 shows the effect of the batch-check size on the

\begin{tabular}{|c|c|c|c|}
\hline Batch-Check Size & \multicolumn{3}{|c|}{ Number of Detected Issues } \\
& must-unsound & $\geq$ 3-unsound & $\geq$ 3-imprecise \\
\hline 1 & 2 & 2 & 71 \\
2 & 6 & 6 & 110 \\
4 & 3 & 5 & 158 \\
8 & 10 & 10 & 193 \\
16 & 9 & 10 & 165 \\
32 & 11 & 22 & 201 \\
\hline
\end{tabular}

Table 7: Effect of batch-check size on the number of detected issues. number of detected issues. Overall, larger batch-check sizes are more effective in detecting soundness and precision issues. During our experiments, we also found that larger batch-check sizes typically help in detecting the same issues faster (that is, with a smaller initial budget) in comparison to smaller sizes.

\section{Threats to Validity}

We have identified the following threats to the validity of our experiments.

Selection of seed programs. Our experimental results may not generalize to other seed programs [43]. However, we evaluated our technique by selecting seed programs from most categories of a well-established repository of verification tasks [7] and by running the program analyzers on tens of thousands of program variants. We, therefore, believe that our benchmark selection significantly helps mitigate this threat and aids generalizability of our results.

Selection of program analyzers. For our experiments, we used the program analyzers described in Sect. V-B. Our findings depend on bugs, unsoundness, and imprecision in these analyzers and, thus, may not generalize. However, our selection includes a wide range of program-analysis techniques, like model checking and abstract interpretation. Moreover, all of these techniques are implemented in mature tools.

Type of checked properties. Our results may also not generalize to other types of checks, for example, for pointsto properties. Our implementation targets numerical safety properties since they are found in almost every program and can, therefore, be checked by most analyzers. Independently, our approach and implementation are configurable and may be extended to also synthesize other types of properties, for instance, by checking if two pointers are aliases.

Randomness in check synthesis. Another potential threat has to do with the internal validity [43] of our experiments, which refers to whether any systematic errors are introduced in the experimental setup. A typical threat to the internal validity of experiments with randomized techniques is the selection of seeds. Recall that our check-synthesis component selects candidate expressions and constants in a randomized way. To ensure deterministic results and to avoid favoring certain program analyzers over others, $\alpha$-Diff uses the same, predefined random seeds for all analyzer configurations.

Survey of developers. A potential threat to the validity of our results is that the survey questions were not understandable or presented in a clear way. To alleviate this concern, we piloted the survey and fine-tuned the questions based on the feedback we received. Moreover, the survey responses might not be representative of other professional $\mathrm{C}$ developers. However, we screened the candidates and excluded any survey participants who did not seem experienced enough.

\section{RELATED WORK}

In the literature, there are several techniques for evaluating different qualities of program analyzers. Especially to ensure soundness of an analyzer, existing work has explored a wide spectrum of techniques requiring varying degrees of human 
effort, for instance, manual proofs (e.g., [44]), interactive and automatic proofs (e.g., [45], [46]), testing (e.g., [47], [48]), and "smoke checking" [13]. There also exist evaluations of the efficiency [49] and precision [50] of various analyses.

Our approach is the first to differentially test real-world program analyzers with the goal of detecting soundness and precision issues in arbitrary code. Specifically, we identify such issues by comparing the results of several analyzers, instead of relying on fixed test oracles.

Testing analyzers with randomly generated programs. Running a program analyzer on randomly generated input programs has proved effective in revealing crashes [51]. However, it is very challenging to randomly generate programs from scratch such that they reveal soundness and precision issues in mature analyzers. Instead, our approach takes as input existing, complex programs as seeds and uses them to generate seed variants by synthesizing checks for numerical properties.

Testing symbolic execution engines. Kapus and Cadar use random program generation in combination with differential testing to find bugs in symbolic execution engines [9], by for instance comparing crashes, output differences, and code coverage. Unlike our approach, this work specifically targets symbolic execution engines and compares the tested engines on randomly generated programs.

Testing abstract interpreters. A common technique for revealing soundness issues in analyzers that infer invariants (e.g., abstract interpreters [38], [24], [25]) is to turn inferred invariants into explicit assertions and then check if the assertions are violated in concrete executions of the program [51], [52], [53]. Concrete executions (e.g., from existing test suites) are also helpful in identifying certain precision issues by observing the effect of intersecting the inferred invariants with concrete runtime values on the number of generated warnings. In contrast, our technique works for any type of safety checker. In addition, if any of the tested analyzers perform an under-approximation (e.g., a bounded model checker or a dynamic symbolic execution engine), our technique essentially compares the results of the other analyzers against a test suite that is automatically generated on the fly.

A usual source of soundness and precision issues in abstract interpreters is bugs in the implementation of the underlying abstract domains and their operations (e.g., intersection and union of abstract states). Existing techniques [47], [54], [48] for detecting such issues use well-known mathematical properties of domains as test oracles. In contrast, our approach can not only detect issues in domain implementations, but also in abstract transformers, which model program statements such as arithmetic operations or method calls.

Evaluating unsoundness in static analyzers. Unsoundness is ubiquitous in static analyzers [15], typically to intentionally favor other important qualities, such as precision or efficiency. A recent technique systematically documents and evaluates the sources of intentional unsoundness in a widely used, commercial static analyzer [16]. The experimental evaluation of this work sheds light on how often the unsoundness of the analyzer causes it to miss bugs. In comparison, our approach treats any tested program analyzer as a black box, and it is also able to detect unintentional soundness and precision issues, caused by bugs in the implementation of the analyzers.

Even more recently, an empirical study evaluated how many known bugs are missed by three industrial-strength static bug detectors [55]. An important difference with our approach is that the checked properties in this study did not necessarily lie within the capabilities of the analyzers. In contrast, we synthesize numerical properties, which should be handled by all analyzers we tested. Moreover, our approach automatically synthesizes potentially erroneous programs and uses differential testing to identify both soundness and precision issues.

Formally verifying program analyzers. To avoid any soundness issues in the first place, interactive theorem provers are often used to verify the soundness of the design of a program analyzer. For instance, this is a common approach for type systems [56], [57]. However, such proofs cannot generally guarantee the absence of soundness issues in the actual implementation of the analyzer. To address this problem, the Verasco [58] project generates the executable code of several abstract domains directly from their Coq formalizations. Even if this approach were more practical, it would still not easily detect precision issues in an analyzer.

Testing compilers. Compilers typically apply different lightweight program analyses (e.g., constant propagation) to produce more efficient code. Existing work [59], [60], [61], [62], [63] has proposed several techniques for detecting bugs in compilers, and indirectly, in their analyses. These techniques often use metamorphic testing to derive test oracles [64], for instance, by comparing the output of two compiled programs where one is a slight, semantics-preserving modification of the other. In contrast, our approach compares several analyzers at once and uses their results to detect soundness and precision issues. In addition, our check synthesis instruments the seed program with assertions that may alter its semantics, for example, by introducing failing executions.

\section{CONCLUSION}

We have proposed a novel and automated technique for differentially testing the soundness and precision of program analyzers. We used it to test six mature, state-of-the-art analyzers on tens of thousands of programs. Our technique found soundness and precision issues in four of these analyzers.

In future work, we plan to explore how to synthesize checks for different types of properties (for instance, hyperproperties [65] like information flow, and liveness properties like termination). We also plan to apply our technique on a larger scale by using safety-critical programs, such as flight controllers, as seed programs with the goal of generating new, challenging verification benchmarks.

\section{ACKNOWLEDGMENTS}

We are very grateful to the program-analysis designers that we contacted, Matthias Dangl, Arie Gurfinkel, Jorge Navas, and Zvonimir Rakamaric, for their prompt replies and fixes as well as for their insightful explanations. 


\section{REFERENCES}

[1] C. Calcagno, D. Distefano, J. Dubreil, D. Gabi, P. Hooimeijer, M. Luca, P. W. O'Hearn, I. Papakonstantinou, J. Purbrick, and D. Rodriguez, "Moving fast with software verification," in NFM, ser. LNCS, vol. 9058. Springer, 2015, pp. 3-11.

[2] C. Calcagno and D. Distefano, "Infer: An automatic program verifier for memory safety of C programs," in NFM, ser. LNCS, vol. 6617. Springer, 2011, pp. 459-465.

[3] P. Godefroid, M. Y. Levin, and D. A. Molnar, "Automated whitebox fuzz testing," in NDSS. The Internet Society, 2008, pp. 151-166.

[4] E. Bounimova, P. Godefroid, and D. A. Molnar, "Billions and billions of constraints: Whitebox fuzz testing in production," in ICSE. IEEE Computer Society/ACM, 2013, pp. 122-131.

[5] B. Blanchet, P. Cousot, R. Cousot, J. Feret, L. Mauborgne, A. Miné, D. Monniaux, and X. Rival, "A static analyzer for large safety-critical software," in PLDI. ACM, 2003, pp. 196-207.

[6] P. Cousot, R. Cousot, J. Feret, L. Mauborgne, A. Miné, D. Monniaux, and X. Rival, "The ASTRÉE analyzer," in ESOP, ser. LNCS, vol. 3444. Springer, 2005, pp. 21-30.

[7] D. Beyer, "Competition on software verification (SV-COMP)," 2017, https://sv-comp.sosy-lab.org.

[8] D. R. Engler, D. Y. Chen, and A. Chou, "Bugs as deviant behavior: A general approach to inferring errors in systems code," in SOSP. ACM, 2001, pp. 57-72.

[9] T. Kapus and C. Cadar, "Automatic testing of symbolic execution engines via program generation and differential testing," in ASE. IEEE Computer Society, 2017, pp. 590-600.

[10] M. Christakis and C. Bird, "What developers want and need from program analysis: An empirical study," in ASE. ACM, 2016, pp. 332 343.

[11] E. M. Clarke, D. Kroening, and F. Lerda, "A tool for checking ANSIC programs," in TACAS, ser. LNCS, vol. 2988. Springer, 2004, pp. $168-176$.

[12] Z. Rakamaric and M. Emmi, "SMACK: Decoupling source language details from verifier implementations," in $C A V$, ser. LNCS, vol. 8559. Springer, 2014, pp. 106-113.

[13] M. Barnett, B.-Y. E. Chang, R. DeLine, B. Jacobs, and K. R. M. Leino, "Boogie: A modular reusable verifier for object-oriented programs," in FMCO, ser. LNCS, vol. 4111. Springer, 2005, pp. 364-387.

[14] D. Giannakopoulou, Z. Rakamaric, and V. Raman, "Symbolic learning of component interfaces," in SAS, ser. LNCS, vol. 7460. Springer, 2012, pp. $248-264$.

[15] B. Livshits, M. Sridharan, Y. Smaragdakis, O. Lhoták, J. N. Amaral, B.Y. E. Chang, S. Z. Guyer, U. P. Khedker, A. Møller, and D. Vardoulakis, "In defense of soundiness: A manifesto," CACM, vol. 58, pp. 44-46, 2015.

[16] M. Christakis, P. Müller, and V. Wüstholz, "An experimental evaluation of deliberate unsoundness in a static program analyzer," in VMCAI, ser. LNCS, vol. 8931. Springer, 2015, pp. 336-354.

[17] D. Beyer and M. E. Keremoglu, "CPAchecker: A tool for configurable software verification," in CAV, ser. LNCS, vol. 6806. Springer, 2011, pp. 184-190.

[18] S. Graf and H. Saïdi, "Construction of abstract state graphs with PVS," in $C A V$, ser. LNCS, vol. 1254. Springer, 1997, pp. 72-83.

[19] T. Ball, R. Majumdar, T. D. Millstein, and S. K. Rajamani, "Automatic predicate abstraction of C programs," in PLDI. ACM, 2001, pp. 203213.

[20] T. A. Henzinger, R. Jhala, R. Majumdar, and G. Sutre, "Lazy abstraction," in POPL. ACM, 2002, pp. 58-70.

[21] A. F. Donaldson, L. Haller, D. Kroening, and P. Rümmer, "Software verification using k-induction," in $S A S$, ser. LNCS, vol. 6887. Springer, 2011, pp. 351-368.

[22] G. Gange, J. A. Navas, P. Schachte, H. Søndergaard, and P. J. Stuckey, "An abstract domain of uninterpreted functions," in VMCAI, ser. LNCS, vol. 9583. Springer, 2016, pp. 85-103.

[23] G. Gange, J. A. Navas, P. Schachte, H. Søndergaard, and P. J. Stuckey, "Exploiting sparsity in difference-bound matrices," in SAS, ser. LNCS, vol. 9837. Springer, 2016, pp. 189-211.

[24] P. Cousot and R. Cousot, "Abstract interpretation: A unified lattice model for static analysis of programs by construction or approximation of fixpoints," in POPL. ACM, 1977, pp. 238-252.

[25] P. Cousot and R. Cousot, "Systematic design of program analysis frameworks," in POPL. ACM, 1979, pp. 269-282.
[26] A. Miné, "Weakly relational numerical abstract domains. (Domaines numériques abstraits faiblement relationnels)," Ph.D. dissertation, École Polytechnique, Palaiseau, France, 2004.

[27] A. Gurfinkel, T. Kahsai, A. Komuravelli, and J. A. Navas, "The SeaHorn verification framework," in CAV, ser. LNCS, vol. 9206. Springer, 2015, pp. 343-361.

[28] A. Komuravelli, A. Gurfinkel, S. Chaki, and E. M. Clarke, "Automatic abstraction in SMT-based unbounded software model checking," in $C A V$, ser. LNCS, vol. 8044. Springer, 2013, pp. 846-862.

[29] A. Komuravelli, A. Gurfinkel, and S. Chaki, "SMT-based model checking for recursive programs," in CAV, ser. LNCS, vol. 8559. Springer, 2014, pp. 17-34.

[30] L. de Moura and N. Bjørner, "Z3: An efficient SMT solver," in TACAS, ser. LNCS, vol. 4963. Springer, 2008, pp. 337-340.

[31] A. R. Bradley, "SAT-based model checking without unrolling," in VMCAI, ser. LNCS, vol. 6538. Springer, 2011, pp. 70-87.

[32] A. Lal, S. Qadeer, and S. K. Lahiri, "A solver for reachability modulo theories," in CAV, ser. LNCS, vol. 7358. Springer, 2012, pp. 427-443.

[33] M. Heizmann, J. Hoenicke, and A. Podelski, "Software model checking for people who love automata," in $C A V$, ser. LNCS, vol. 8044. Springer, 2013, pp. 36-52.

[34] D. Dietsch, M. Heizmann, B. Musa, A. Nutz, and A. Podelski, "Craig vs. Newton in software model checking," in ESEC/FSE. ACM, 2017, pp. 487-497.

[35] M. Heizmann, Y. Chen, D. Dietsch, M. Greitschus, J. Hoenicke, Y. Li, A. Nutz, B. Musa, C. Schilling, T. Schindler, and A. Podelski, "Ultimate Automizer and the search for perfect interpolants-(competition contribution)," in TACAS, ser. LNCS, vol. 10806. Springer, 2018, pp. $447-451$.

[36] A. Miné, "The Octagon Abstract Domain," HOSC, vol. 19, pp. 31-100, 2006.

[37] P. Cousot and N. Halbwachs, "Automatic discovery of linear restraints among variables of a program," in POPL. ACM, 1978, pp. 84-96.

[38] P. Cousot and R. Cousot, "Static determination of dynamic properties of programs," in ISOP. Dunod, 1976, pp. 106-130.

[39] P. Cousot and R. Cousot, "Comparing the Galois connection and widening/narrowing approaches to abstract interpretation," in PLILP, ser. LNCS, vol. 631. Springer, 1992, pp. 269-295.

[40] D. Monniaux and J. Le Guen, "Stratified static analysis based on variable dependencies," ENTCS, vol. 288, pp. 61-74, 2012.

[41] G. Amato and M. Rubino, "Experimental evaluation of numerical domains for inferring ranges," ENTCS, vol. 334, pp. 3-16, 2018.

[42] C. Lattner and V. S. Adve, "LLVM: A compilation framework for lifelong program analysis \& transformation," in $C G O$. IEEE Computer Society, 2004, pp. 75-88.

[43] J. Siegmund, N. Siegmund, and S. Apel, "Views on internal and external validity in empirical software engineering," in ICSE. IEEE Computer Society, 2015, pp. 9-19.

[44] J. Midtgaard, M. D. Adams, and M. Might, "A structural soundness proof for Shivers's escape technique: A case for Galois connections," in SAS, ser. LNCS, vol. 7460. Springer, 2012, pp. 352-369.

[45] F. Besson, P.-E. Cornilleau, and T. P. Jensen, "Result certification of static program analysers with automated theorem provers," in VSTTE, ser. LNCS, vol. 8164. Springer, 2013, pp. 304-325.

[46] S. Blazy, V. Laporte, A. Maroneze, and D. Pichardie, "Formal verification of a $\mathrm{C}$ value analysis based on abstract interpretation," in $S A S$, ser. LNCS, vol. 7935. Springer, 2013, pp. 324-344.

[47] J. Midtgaard and A. Møller, "QuickChecking static analysis properties," Softw. Test., Verif. Reliab., vol. 27, 2017.

[48] A. Bugariu, V. Wüstholz, M. Christakis, and P. Müller, "Automatically testing implementations of numerical abstract domains," in ASE. ACM, 2018, pp. 768-778.

[49] M. Sridharan and S. J. Fink, "The complexity of Andersen's analysis in practice," in SAS, ser. LNCS, vol. 5673. Springer, 2009, pp. 205-221.

[50] P. Liang, O. Tripp, M. Naik, and M. Sagiv, "A dynamic evaluation of the precision of static heap abstractions," in OOPSLA. ACM, 2010, pp. 411-427.

[51] P. Cuoq, B. Monate, A. Pacalet, V. Prevosto, J. Regehr, B. Yakobowski, and $\mathrm{X}$. Yang, "Testing static analyzers with randomly generated programs," in NFM, ser. LNCS, vol. 7226. Springer, 2012, pp. 120-125.

[52] J. Wu, G. Hu, Y. Tang, and J. Yang, "Effective dynamic detection of alias analysis errors," in ESEC/FSE. ACM, 2013, pp. 279-289. 
[53] E. S. Andreasen, A. Møller, and B. B. Nielsen, "Systematic approaches for increasing soundness and precision of static analyzers," in SOAP. ACM, 2017, pp. 31-36.

[54] M. Madsen and O. Lhoták, "Safe and sound program analysis with FLIX," in ISSTA. ACM, 2018, pp. 38-48.

[55] A. Habib and M. Pradel, "How many of all bugs do we find? A study of static bug detectors," in ASE. ACM, 2018, pp. 317-328.

[56] C. Dubois, "Proving ML type soundness within Coq," in TPHOLs, ser. LNCS, vol. 1869. Springer, 2000, pp. 126-144.

[57] Z. Shao, B. Saha, V. Trifonov, and N. Papaspyrou, "A type system for certified binaries," in POPL. ACM, 2002, pp. 217-232.

[58] J. Jourdan, V. Laporte, S. Blazy, X. Leroy, and D. Pichardie, "A formallyverified C static analyzer," in POPL. ACM, 2015, pp. 247-259.

[59] X. Yang, Y. Chen, E. Eide, and J. Regehr, "Finding and understanding bugs in C compilers," in PLDI. ACM, 2011, pp. 283-294.

[60] V. Le, M. Afshari, and Z. Su, "Compiler validation via equivalence modulo inputs," in PLDI. ACM, 2014, pp. 216-226.

[61] V. Le, C. Sun, and Z. Su, "Finding deep compiler bugs via guided stochastic program mutation," in OOPSLA. ACM, 2015, pp. 386-399.

[62] C. Lidbury, A. Lascu, N. Chong, and A. F. Donaldson, "Many-core compiler fuzzing," in PLDI. ACM, 2015, pp. 65-76.

[63] C. Sun, V. Le, and Z. Su, "Finding and analyzing compiler warning defects," in ICSE. ACM, 2016, pp. 203-213.

[64] E. T. Barr, M. Harman, P. McMinn, M. Shahbaz, and S. Yoo, "The oracle problem in software testing: A survey," TSE, vol. 41, pp. 507-525, 2015.

[65] M. R. Clarkson and F. B. Schneider, "Hyperproperties," in CSF. IEEE Computer Society, 2008, pp. 51-65. 